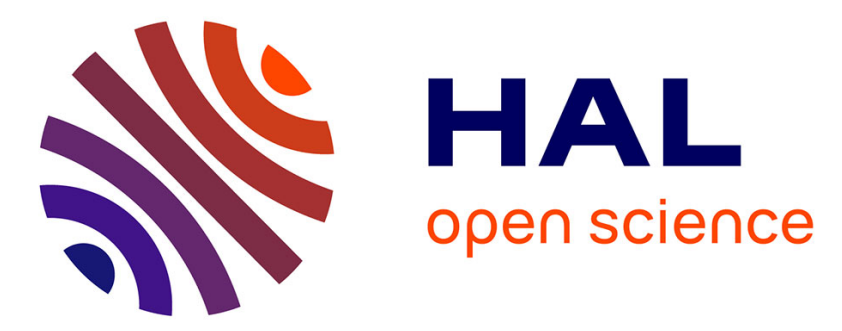

\title{
Photoionization microscopy on magnesium atom and comparison with hydrogenic theory
}

P. Kalaïtzis, S. Danakas, F. Lepine, C. Bordas, S. Cohen

\section{To cite this version:}

P. Kalaïtzis, S. Danakas, F. Lepine, C. Bordas, S. Cohen. Photoionization microscopy on magnesium atom and comparison with hydrogenic theory. 10th Jubilee International Conference of the Balkan Physical Union, Aug 2018, Sofia, Bulgaria. pp.050004, 10.1063/1.5091172 . hal-02346217

\section{HAL Id: hal-02346217 \\ https://hal.science/hal-02346217}

Submitted on 7 Nov 2019

HAL is a multi-disciplinary open access archive for the deposit and dissemination of scientific research documents, whether they are published or not. The documents may come from teaching and research institutions in France or abroad, or from public or private research centers.
L'archive ouverte pluridisciplinaire HAL, est destinée au dépôt et à la diffusion de documents scientifiques de niveau recherche, publiés ou non, émanant des établissements d'enseignement et de recherche français ou étrangers, des laboratoires publics ou privés. 


\section{Photoionization microscopy on magnesium atom and comparison with hydrogenic theory}

Cite as: AIP Conference Proceedings 2075, 050004 (2019); https://doi.org/10.1063/1.5091172

Published Online: 26 February 2019

P. Kalaitzis, S. Danakas, F. Lépine, C. Bordas, and S. Cohen
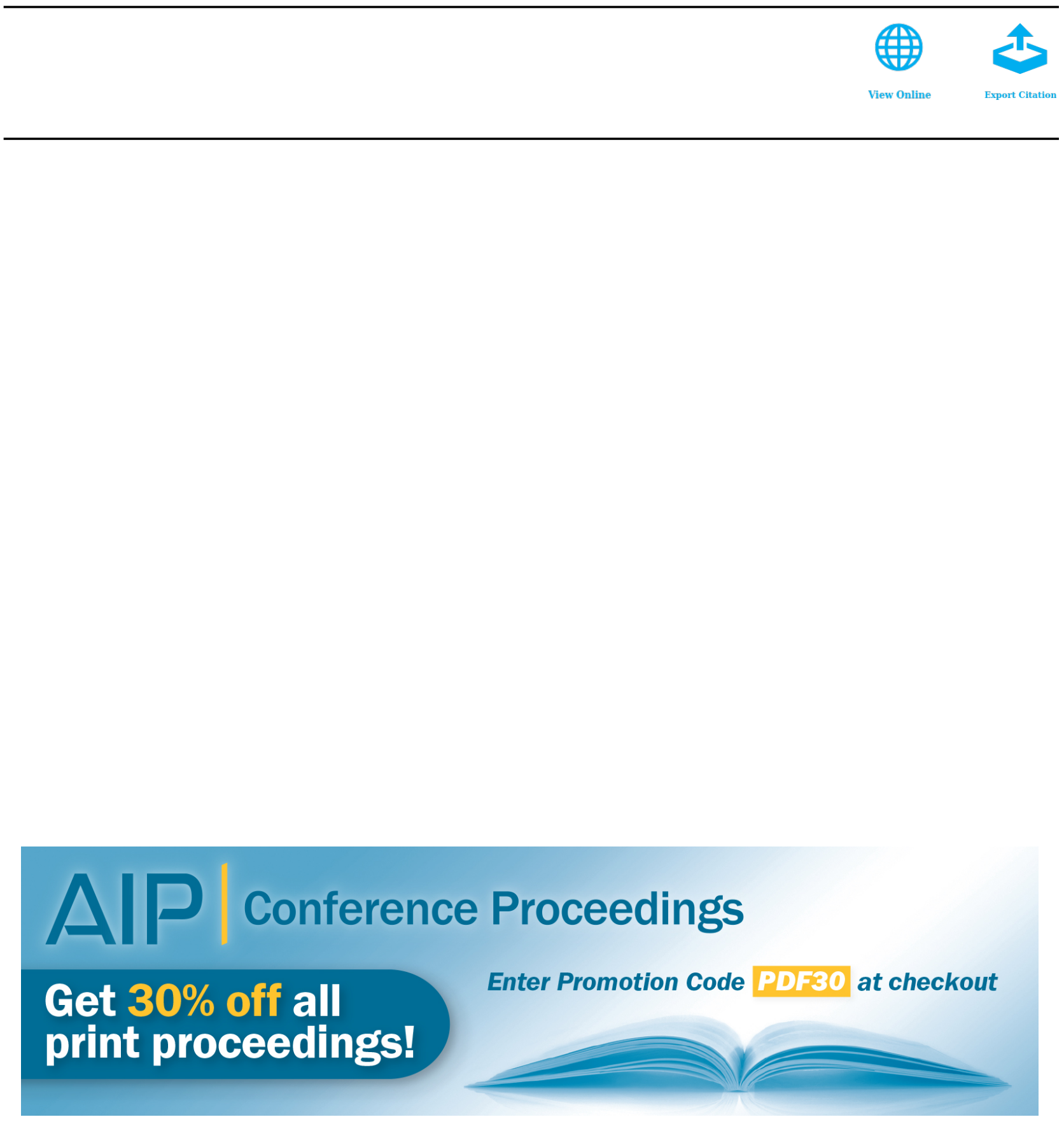


\title{
Photoionization Microscopy on Magnesium Atom and Comparison with Hydrogenic Theory
}

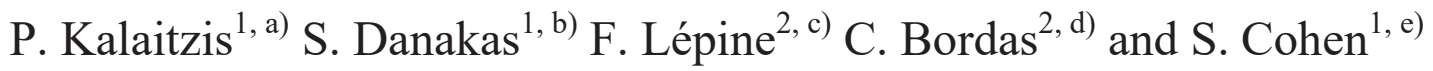 \\ ${ }^{1}$ Atomic and Molecular Physics Laboratory, Physics Department, University of Ioannina, 45110 Ioannina, Greece \\ ${ }^{2}$ Institut Lumière Matière, Université Lyon 1, CNRS, UMR 5306, 10 rue Ada Byron 69622 Villeurbanne Cedex, \\ France

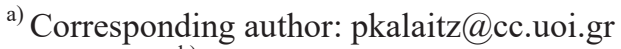 \\ b) sdanakas@uoi.gr \\ c) franck.lepine@univ-lyon1.fr \\ d) cbordas@univ-lyon1.fr \\ e) scohen@uoi.gr
}

\begin{abstract}
The experimental technique of photoionization microscopy (PM) allows for the measurement of the slow $(\mathrm{meV})$ electron current probability density in the case of atomic photoionization in the presence of a static electric field. The spatial structure of the electron flux (differential cross section), as imaged on a plane detector perpendicular to the field, exhibits a radial and an angular dependence. Here attention is paid to the radial distribution, obtained after angularly integrating the electron flux. We discuss the energy evolution of this quantity in the form of a two-dimensional contour map with the abscissa being the outgoing electron energy and the ordinate being the radius on the detector. As a working example we employ the experimental map recorded during a PM study of the Mg atom. Near-threshold the map reveals quite complex quantum interference and beating patterns. Many of these patterns are also observed in a theoretical map concerning the hydrogen atom and calculated by employing a non-perturbative quantum mechanical treatment. Similarities between the maps which are of "universal" nature are identified, while the observed differences are attributed to the different target atoms, initial states and excitation schemes. Finally, possible applications and directions of further work are also briefly discussed.
\end{abstract}

\section{INTRODUCTION}

Photoionization microscopy (PM) relies on the recording of the two-dimensional flux of very slow (meV) electrons ejected during the photoionization of neutral atoms in the presence of a uniform static electric field. The flux is recorded by a position sensitive detector and under certain conditions the obtained image exhibits quantum interference effects which directly reflect the squared modulus of the electronic wave-function $[1,2,3,4]$. The term "microscopy" is justified because, along the direction of the static field, the Stark wave-function of the outgoing photoelectron extends over macroscopically large distances (while being bound transversally to it). Moreover, just above the field-modified ionization threshold continuum and quasi-bound Stark states coexist and, therefore, PM may provide access to the wave-functions of either of them. 
The PM concept was introduced during the early 1980s [1,5,6,7] within the framework of the hydrogenic Stark effect. Experimental studies were up to now devoted to hydrogen itself [8] and to the light Li and He atoms [9,10,11 ] (quasi-bound state wave functions), and to the heavy Xe atom [12] (continuum wave functions). Here we employ as target the medium complexity magnesium atom and explore phenomena having their origin in the exclusive presence of the continua. In other words, the purpose of this work is to seek interferences of "universal" nature; the latter revealed by a comparison of our measurements with hydrogenic quantum mechanical calculations. Finally, the conclusions of our discussion will also point towards the directions of further experimental and theoretical work.

\section{THEORETICAL FRAMEWORK OF PHOTOIONIZATION MICROSCOPY}

Our experimental data will be compared below with fully quantum mechanical calculations [13]. Nevertheless, the description of PM in classical and semiclassical terms would be probably more instructive for the reader. Therefore, we highlight in this section the classical $[14,15]$ and semiclassical $[1,2,16,17]$ treatment of the relevant Coulomb-Stark problem. Particularly, it is our aim here to provide those elements that are necessary to identify the main features of a PM image.

Let an electron move under the influence of both a static electric field $\mathbf{F}=F \mathbf{z}$ and the Coulomb attraction (structure less ion with $Z=1$ ). For this problem the azimuthal number $m$ remains a good quantum number $[7,18]$. Below the discussion will be restricted to the $m=0$ case. Further, it is advantageous to introduce the reduced energy parameter $\varepsilon=E /\left|E_{\mathrm{sp}}\right|$, where $E$ the electron's energy and $E_{\mathrm{sp}}=-2 F^{1 / 2}$ the saddle point energy. For $E<-\left|E_{\mathrm{sp}}\right|(\varepsilon<-1)$ the electron is trapped and can never escape (ionize) from the attractive Coulomb center. Therefore, in photoionization studies we are interested exclusively for the $\varepsilon>-1$ energy range. Assume that the electron is initially at the axis origin and that it is launched with an angle $\beta$ with respect to the positive $z$ axis. $\beta=0$ denotes uphill ejection while $\beta=\pi$ denotes downhill ejection towards a detector placed at some distance $z_{\text {det }}<0$. Within the $-1<\varepsilon<0$ energy range a critical ejection angle $\beta_{\mathrm{c}}$ [14] separates the bound from the unbound motion. For $\beta<\beta_{\mathrm{c}}$ the electron is trapped and does not ionize (despite the fact that this is energetically possible), while for $\beta>\beta_{\mathrm{c}}$ the electron escapes to infinity. For positive energies $\beta_{\mathrm{c}}$ loses its meaning and the electron can reach the detector's plane for every ejection angle $\beta$.

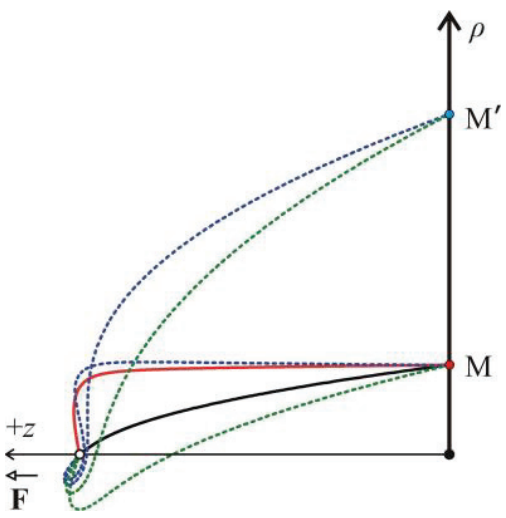

(a)

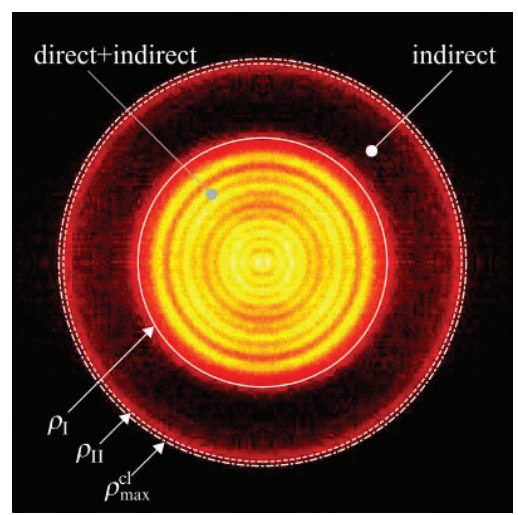

(b)

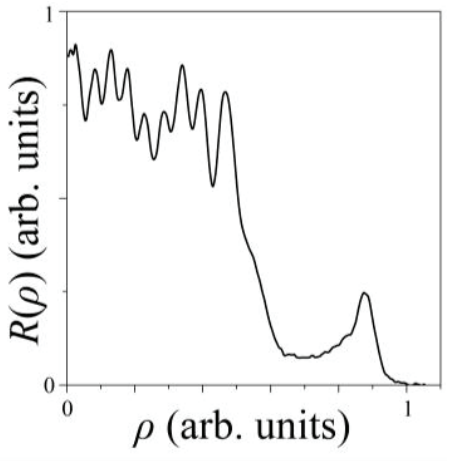

(c)

FIGURE 1. (a) Classical electron trajectories for different ejection angles $\beta>\beta_{\mathrm{c}}$ and $\varepsilon=-0.16, F=680 \mathrm{~V} / \mathrm{cm}$. The Coulomb center (hollow point) is placed at $z=\rho=0$ where $\rho=0$ denotes the center of the detector plane (bold vertical line). Point M can be reached by both direct (solid lines) and indirect (dashed lines) trajectories while $\mathrm{M}^{\prime}$ only by the indirect ones. Several more indirect curves end up to $\mathrm{M}$ and $\mathrm{M}^{\prime}$ but they are omitted for the sake of clarity. (b) Experimental magnesium PM image in color scale for $\varepsilon \approx-0.16, F=680 \mathrm{~V} / \mathrm{cm}$ and $m=0$. The following maximum radii are also shown: $\rho_{\text {I }}$ (white solid line), $\rho_{\text {II }}$ (white dashed line) and $\rho_{\max }^{\mathrm{cl}}$ (white dashed dotted line). (c) Radial distribution $R(\rho)$ computed from the image in (b). Note the modulation of the finer fringes caused by the beating between electron waves corresponding to the direct and indirect trajectories.

Figure 1(a) shows schematically sample trajectories reaching a detector for $\varepsilon=-0.16$ and $F=680 \mathrm{~V} / \mathrm{cm}$. From the plot one can distinguish between two classes of trajectories: (a) Those which never intersect the $z$ axis (hereafter called direct trajectories) and are almost parabolic (solid lines). The existence of these trajectories is possible solely above a critical energy $\varepsilon_{\mathrm{dir}} \approx-0.755[14,16]$. (b) Those trajectories which intersect at least once the $z$ axis (dashed 
lines). These indirect trajectories are quite complex, due to the fact that the electron is multiply re-scattered by the residual ion before it finally escapes to infinity.

Each ejection angle $\beta$ is associated with a given trajectory and a given point of impact $\mathrm{M}$ at distance $\rho$ from the detector's center. This generalized classical scattering deflection function $\rho(\beta)[14,19]$ generally exhibits several maxima and several zeros. The maxima are denoted as $\rho_{\mathrm{I}}, \rho_{\mathrm{II}}, \ldots$ etc, where $\rho_{\mathrm{I}}$ is entirely related to the direct trajectories. All other maxima are related to indirect trajectories but in practice they are all indistinguishable from $\rho_{\text {II }}$ and can be approximated by the classical maximum radius $\rho_{\max }^{\mathrm{cl}}$ which is given by a simple analytical expression [14]. It also holds that $\rho_{\mathrm{I}}<\rho_{\mathrm{II}}<\ldots<\rho_{\max }^{\mathrm{cl}}$. Further, all these maximum radii progressively merge together for high positive energies. Figure 1(b) presents a Mg experimental PM image, recorded at $\varepsilon \approx-0.16$. The indirect trajectories contribution spans the range $0 \leq \rho \leq \rho_{\text {II }}$ and forms the outer bright ring at $\rho \approx \rho_{\text {II }}$ while the radii of the direct trajectories extend over the $0 \leq \rho \leq \rho_{\mathrm{I}}$ range. Finally, the several zeros of $\rho(\beta)$ give rise to an intense peak at the center of the image which is typically referred as the glory signal [19].

As it is evident in Figure 1(a) there are numerous trajectories connecting the electron's origin with a given detector point. This fact implies the emergence of quantum interference effects that can be taken into account semiclassically $[1,16]$. Within such an approach, the phase accumulated along a given pathway is calculated by integrating the electron momentum over the full trajectory. The final interferogram is proportional to the squared modulus of the coherent sum of the participating electron waves which are associated with a given detector point. The interference is controlled by the phase differences of these waves, reflecting the respective path length differences. The $\rho_{\mathrm{I}} \leq \rho \leq \rho_{\mathrm{II}}$ detector zone may be reached by indirect trajectories only and the associated fringe system reflects interferences attributed exclusively to this type of trajectories (see point $\mathbf{M}^{\prime}$ of Figure 1(a)). This fringe system is not visible in the Mg image of Figure 1(b) due to its weak magnitude and the small successive fringe spacing corresponding to this energy $(\varepsilon=-0.16)$, which is incompatible with the spatial resolution of the detector. On the other hand, the $0<\rho<\rho_{\text {I }}$ zone is characterized by the presence of both direct and indirect contributions (see point $\mathrm{M}$ of Figure 1(a)). The main contribution stems from the much more intense direct fringe system. Nevertheless, the existence of indirect trajectories affects the interferogram, giving notably rise to important beating effects. These effects persist at least up to $\varepsilon \approx+1$ [13] and they are more easily observed in the radial distribution $R(\rho)$, obtained by angularly integrating the image with respect to its center (see Figure 1(c)).

\section{EXPERIMENTAL SET-UP AND PROCEDURE}

The experimental set-up used for the magnesium measurements has been recently exposed in some detail [13] so here we provide just a brief description. Coherent radiation is produced by a dye laser pumped by the second harmonic $(532 \mathrm{~nm})$ of a Nd:YAG laser, operating at a repetition rate of $10 \mathrm{~Hz}$. The dye laser operates within the $610-670 \mathrm{~nm}$ wavelength range and its linewidth is $\sim 0.2 \mathrm{~cm}^{-1}$, while its pulses have $\sim 5 \mathrm{~ns}$ duration, and $\sim 10 \mathrm{~mJ}$ energy. For the excitation of $\mathrm{Mg}$ we frequency-double the dye laser radiation by means of a KDP crystal. The pulse energy of the frequency-doubled pulses is $\sim 1 \mathrm{~mJ}$. The fundamental $(\omega, \mathrm{VIS})$ and frequency-doubled $(2 \omega, \mathrm{UV})$ beams are separated by an appropriate filter, reflecting the $\omega$-beam and transmitting the $2 \omega$-one. The $\omega$-beam is guided to a wavelength calibration system. The latter consists of a $\mathrm{Ne}$ optogalvanic lamp providing absolute calibration via the one-photon spectrum of Ne and a Fabry-Perot interferometer (free spectral range $0.4729(2) \mathrm{cm}^{-1}$ ) that allows for relative calibration. The linear polarization of the UV beam is controlled by means of a linear polarizer and a double Fresnel rhomb, acting as an achromatic $\lambda / 2$ retarder.

The $\mathrm{Mg}$ atomic vapor is produced in an electrically heated stainless-steel oven. A $1 \mathrm{~mm}$ hole separates the oven from a main chamber into which the atoms expand and a thermal atomic beam is formed. The main chamber is pumped by a turbo-molecular-pump/rotary-pump system and a liquid nitrogen cryo-trap. The achieved background pressure under working conditions is $\sim 10^{-7}$ mbar. The UV-laser beam is focused with a $20 \mathrm{~cm}$ focal length lens in the main chamber where it crosses perpendicularly the $\mathrm{Mg}$ beam. The photoionization of $\mathrm{Mg}$ atoms takes place in between the first two electrodes of a three-electrode velocity-map imaging (VMI) spectrometer [20]. These electrodes are the Repeller (applied voltage $V_{R}$ ) and the Extractor one (applied voltage $V_{E}$ ). The Repeller is solid, while the Extractor has a hole at its center. They are followed by a third grounded electrode whose design is identical to that of the Extractor. This geometry produces an inhomogeneous electric field, which, however, may be considered as nearly constant within the limited laser/atom interaction region. The fulfillment of the VMI condition 
is achieved for a specific $V_{E} / V_{R}$ ratio, characteristic of a given geometry [21]. The field accelerates the electrons towards the detector. They travel along a field-free drift tube and an electrostatic magnifying Einzel lens placed approximately midway this tube [21,22]. The role of this lens is to magnify the recorded images and it consists of three identical and equally-spaced electrodes with holes at their centers. The middle electrode is biased to the voltage $\mathrm{V}_{\mathrm{L}}$ and the two outer electrodes are grounded.

The two-dimensional position-sensitive detector is placed at the end of the field-free drift tube. It consists of a two-microchannel plate assembly followed by a phosphor screen. A CCD camera records the bright spots on this screen and the recorded images are transferred to a PC. After accumulating over several thousand laser shots the 2D distribution of electron impacts is obtained.

Typically, in our experiments the applied electric field strengths range between 500 and $1000 \mathrm{~V} / \mathrm{cm}$, while a $\sim 20$-fold image magnification is achieved. For $\sim 20 \mathrm{meV}$ electrons such a magnification leads to typical image sizes of $\sim 20 \mathrm{~mm}$, i.e. well above the resolution of our camera. The entire spectrometer is shielded, especially near the first three electrodes, by a double $\mu$-metal layer, resulting to a residual magnetic field $<1 \mu \mathrm{T}$ in its interior.

\section{RESULTS AND DISCUSION}

In the present work we focus on PM radial distributions near the zero-field ionization threshold of magnesium atom. Specifically, ground state $\left(3 \mathrm{~s}^{2}{ }^{1} \mathrm{~S}_{0}\right) \mathrm{Mg}$ atoms are excited to the final $3 \mathrm{~s} E k$ Stark states after absorbing twoUV-photons. The laser beam linear polarization is parallel to the static electric field axis. Therefore, due to the relevant $\Delta m=0$ selection rule only $m=0$ final states are excited and the recorded images (like the one in Figure 1(b)) show no angular distribution. The radial distributions $R(\rho)$ are obtained by angularly integrating each image with respect to its center.

The images were recorded using an energy step of $\Delta E \simeq 0.4 \mathrm{~cm}^{-1}$. The full set of obtained radial distributions is presented as a contour map in Figure 2(a), where the horizontal axis is the electron energy with respect to the zerofield $3 \mathrm{~s}_{1 / 2}$ threshold and the vertical axis refers to the radius of impact. In fact, all radii are scaled to the maximum classical radius at the zero-field threshold, $\rho_{\max }^{\mathrm{cl}}(E=0)$. Note that at $E \approx 0 \rho_{\max }^{\mathrm{cl}}(E)$ is practically indistinguishable from the maximum indirect contribution radius $\rho_{\mathrm{II}}(E)$. The measurement lasted for several days and the laser output gradually drifted towards to somewhat lower pulse energies. This drift was imprinted on the electron signal and for avoiding misinterpretations of the data caused by this effect Figure 2(a) actually presents the experimental radial distribution divided by the total electron signal (proportional to the total ionization cross section $\sigma_{\text {tot }}(E)$ ) of each image. We have verified that, in the absence of sharp resonances, occurring in Mg solely near the saddle point energy $E_{\mathrm{sp}}$, this procedure does not alter the characteristics of the map.

The map exhibits rich interference fringe structures, the gross features of which can afford a simple semiclassical description [16,17]. For the discussion of the fine details of the map, however, a quantum treatment of the problem is required. To this purpose we present in Figure 2(b) a fully quantum calculation for the hydrogen atom and for the same field strength and energy range. The framework of the calculation has been described in detail in [13]. The experimental and theoretical maps present a great number of similarities, despite the fact that they refer to different atoms as well as to different excitation schemes. Specifically, each map can be separated in three zones whose borders are roughly predicted by the maximum indirect radius $\rho_{\mathrm{II}} \simeq \rho_{\max }^{\mathrm{cl}}$ and the maximum direct radius $\rho_{\mathrm{I}}$ : (i) The zone comprising the upper part of the maps $\left(\rho>\rho_{\text {III }}\right)$ where there is practically no signal at all, (ii) the zone within the $\rho_{\mathrm{I}}<\rho<\rho_{\text {II }}$ range, which is of moderate magnitude (seen with red colors on the logarithmic scale of the contour maps), and (iii) the small radius $\left(0<\rho<\rho_{\mathrm{I}}\right)$, most intense zone showing quite complex interferences. Our quantum calculations predict the absence of sharp direct-indirect boarder, a feature that is also verified experimentally. However, this border becomes sharper as energy increases and it gradually becomes indistinguishable from $\rho_{\mathrm{I}}$, which, in turn, progressively approaches $\rho_{\text {II }}$.

Let us first discuss the $\rho_{\mathrm{I}}<\rho<\rho_{\text {II }}$ zone. Within this zone only indirect trajectories contribute and this is expected to lead to a simple radial fringe pattern (i.e. fringes along the radius $\rho$, showing little or no beating effects). This is quite evident in the theoretical calculation (Figure 2(b)) but it is absent from the experimental data (Figure 2(a)). The latter can be partially explained by the limited spatial resolution of our position sensitive detector. However, spatial resolution limitations cannot explain the observed absence of radial fringes also at lower energy $(\varepsilon \sim-1)$ [13], which

appears to be a characteristic of two-photon excitation of $\mathrm{Mg}$ ground state. On the other hand, a common feature of the experimental and the theoretical map is the signal magnitude modulation along the energy axis, resulting to 
clearly visible quasi-vertical fringes. The modulation "frequency" $1 / \Delta E$ of these fringes is expected to be field dependent, a fact that may have implications for fairly accurate estimations of the field strength. Finally, particularly interesting is the practically complete suppression of the indirect signal strength, occurring in both the experimental and the theoretical map but at different energy locations: At $\varepsilon \sim-0.35$ in Figure 2(a) and at $\varepsilon \sim-0.15$ in Figure 2(b). This effect is not yet fully understood and it is still under study. It nevertheless appears to be strongly dependent upon the particular target atom, initial state and excitation scheme.

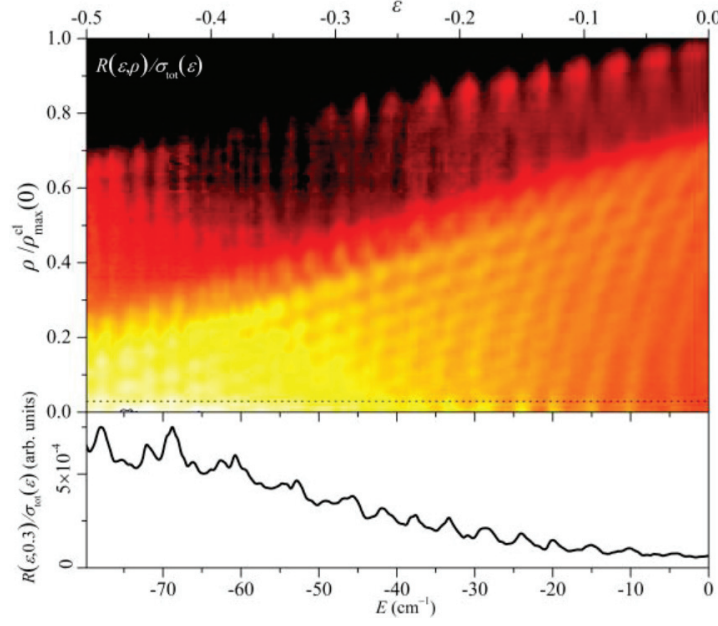

(a)

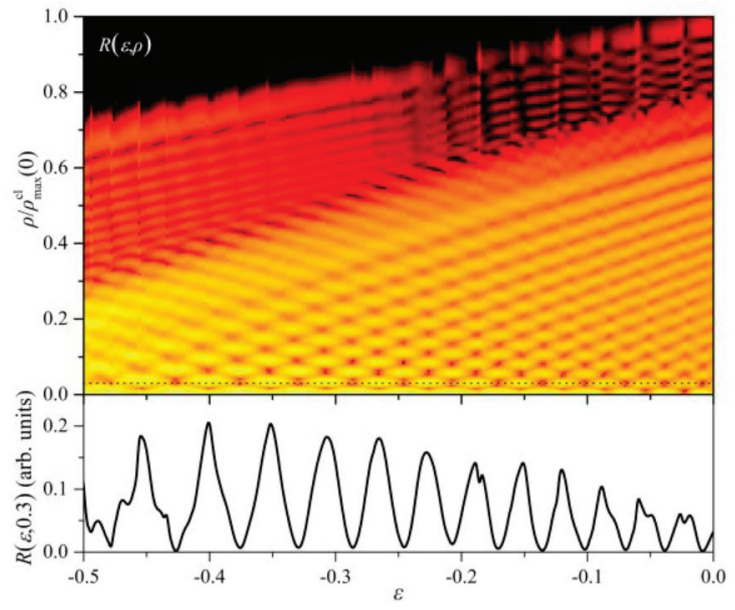

(b)

FIGURE 2. (a) Experimental radial distribution contour map for two-photon excitation of the $\mathrm{Mg}$ ground state $(m=0$ final Stark states). The map magnitude is scaled to the total electron signal, $\sigma_{\mathrm{tot}}$, and the radius is scaled to $\rho_{\max }^{\mathrm{cl}}(E=0)$. The experimental field strength of $F=680 \pm 10 \mathrm{~V} / \mathrm{cm}$ is estimated as explained in [13]. The graph at the bottom of the map shows the linear scale magnitude profile $R(\varepsilon, 0.3) / \sigma_{\text {tot }}(\varepsilon)$. (b) Calculated radial distribution contour map for single-photon excitation of $\mathrm{H}$ out of the $>=\left[|2 \mathrm{~s}>-| 2 \mathrm{p}_{m=0}>\right] / \sqrt{2}$ ( $m=0$ final Stark states). The graph at the bottom of the map shows the linear scale magnitude profile $R(\varepsilon, 0.3)$, exactly on the first radius of strong magnitude modulation. Both contour plots are in logarithmic scale, spanning three orders of magnitude.

We now deal with the more interesting $0<\rho<\rho_{\mathrm{I}}$ zone, where both, direct and indirect, classes of trajectories and consequently fringe systems contribute with appreciable amplitude. In fact, it is expected that the direct contribution will form a strong fringe system that will be modulated by the indirect one. Indeed, this modulation was experimentally observed recently in hydrogen atom [17] and the modulated beating pattern is found to have the form of a checkerboard structure. This structure is also evident in both maps of Figure 2, for the energy range, $-0.5<\varepsilon<-$ 0.1. Furthermore, in the experimental map of Figure 2(a) the checkerboard structure evolves at higher energies to a strong beating effect, in the form of curved quasi-vertical lines superimposed to the direct fine fringe system. The visual effect of this beating can be also noticed in the single image in Figure 1(b).

The above mentioned beating effects seem to be connected to another striking oscillating feature, i.e. the magnitude modulation observed as a function of energy at zero radius $(\rho=0)$. In terms of scattering terminology [19] this so-called glory signal is a consequence of a focusing effect experienced by the electrons due to the presence of the Coulomb center. Since the glory signal is typically the brightest part of the image at this energy range, the modulation can be easily recorded even with experimental set-ups of lower resolution than the present one [15]. The glory signal is evident in both the experimental and the theoretical data of Figure 2 and suggests the study of beating structures in the maps at constant, possibly non-zero, radii. As an example, the graphs at the bottom of the maps of Figure 2 show the magnitude profiles for $\rho / \rho_{\max }^{\mathrm{cl}}=0.3$. The oscillations are evident in both $\mathrm{Mg}$ and $\mathrm{H}$. There are energy ranges where they exhibit a purely sinusoidal behavior, while there are other energy ranges showing a more complex structure (as for example in $\mathrm{Mg}$ near the checkerboard range). Recently these magnitude oscillations were connected to time delays between pairs of electron trajectories, by using the Eisenbud-Wigner time delay definition [23]. Our Mg results imply that the time delay extraction along the lines suggested in [17] may be applicable to the multielectron case as well. 


\section{CONCLUSION}

We have presented a photoionization microscopy study on the $\mathrm{Mg}$ atom, focusing on the evolution of the recorded radial distributions with excitation energy. The experimentally obtained $R(E, \rho)$ map exhibits rich interference structures, including important beating effects. An attempt was made to reveal the map features of "universal" character. To this purpose the experimental map has been compared with the results of a fully quantum mechanical calculation, referring to hydrogen atom and particularly to its single-photon excitation to the final $m=0$ Stark states out of its excited $\left[|2 \mathrm{~s}>-| 2 \mathrm{p}_{m=0}>\right] / \sqrt{2}$ initial state. In general the $R(E, \rho)$ embodies much richer information than the total ionization cross section, as for example time information between electron trajectories. It also reveals distinct patterns that are not visible at individual images. Each of these patterns may provide different pieces of information about the system under study or the excitation process. It, therefore, appears that further experimental and theoretical studies to other multi-electron atoms are in order. This way important phenomena which are specific to a given complex target atom may be revealed. We are currently working along these lines.

\section{ACKNOWLEDGMENTS}

The experiment was performed at the Central Laser Facility of the University of Ioannina. Authors acknowledge support of this work by the project "ELI - LASERLAB Europe Synergy, HiPER \& IPERION-CH.gr" (MIS 5002735) which is implemented under the Action "Reinforcement of the Research and Innovation Infrastructure", funded by the Operational Programme "Competitiveness, Entrepreneurship and Innovation" (NSRF 2014-2020) and co-financed by Greece and the European Union (European Regional Development Fund).

\section{REFERENCES}

1. V. D. Kondratovich and V. N. Ostrovsky, J. Phys. B 23, 3785 (1990).

2. L. B. Zhao and J. B. Delos, Phys. Rev. A 81, 053417 (2010).

3. L. B. Zhao and J. B. Delos, Phys. Rev. A 81, 053418 (2010).

4. F. Texier, Phys. Rev. A 71, 013403 (2005).

5. I. I. Fabrikant, JETP 52, 1045 (1980).

6. Yu. N. Demkov, V. D. Kondratovich, and V. N. Ostrovsky, JETP Lett. 34, 403 (1981).

7. V. D. Kondratovich and V. N. Ostrovsky, J. Phys. B 17, 1981 (1984); J. Phys. B 17, 2011 (1984); J. Phys. B 23, 21 (1990).

8. A. S. Stodolna et al, Phys. Rev. Lett. 110, 213001 (2013).

9. S. Cohen et al, Phys. Rev. Lett. 110, 183001 (2013).

10. S. Cohen et al, Phys. Rev. A 94, 013414 (2016).

11. A. S. Stodolna et al, Phys. Rev. Lett. 113, 103002 (2014).

12. C. Nicole, H. L. Offerhaus, M. J. J. Vrakking, F. Lépine and C. Bordas, Phys. Rev. Lett. 88, 133001 (2002); C. Bordas, F. Lépine, C. Nicole and M. J. J. Vrakking, Phys. Rev. A 68, 012709 (2003); F. Lépine, C. Bordas, C. Nicole and M. J. J. Vrakking, Phys. Rev. A 70, 033417 (2004).

13. P. Kalaitzis, S. Danakas, F. Lépine, C. Bordas and S. Cohen, Phys. Rev. A 97, 053412 (2018).

14. C. Bordas, Phys. Rev. A 58, 400 (1998).

15. T. Barillot et al, J. Chem. Phys. 147, 013929 (2017).

16. C. Bordas, F. Lépine, C. Nicole and M. J. J. Vrakking, Phys. Rev. A 68, 012709 (2003).

17. A. S. Stodolna, F. Lépine, A. Rouzée, S. Cohen, A. Gijsbertsen, J. H. Jungmann-Smith, C. Bordas and M. J. J. Vrakking, J. Phys. B 50, 164001 (2017).

18. T. F. Gallagher, Rydberg Atoms (Cambridge University Press, Cambridge, 1994).

19. S. Cohen, P. Kalaitzis, S. Danakas, F. Lépine and C. Bordas, J. Phys. B 50, 065002 (2017).

20. A. T. J. B. Eppink and D. H. Parker, Rev. Sci. Instrum. 68, 3477 (1997).

21. M. M. Harb, S. Cohen, E. Papalazarou, F. Lépine and C. Bordas, Rev. Sci. Instrum. 81, 125111 (2010).

22. H. L. Offerhaus et al, Rev. Sci. Instrum. 72, 3245 (2001).

23. R. Gaillac, M. Vacher, A. Maquet, R. Taïeb and J. Caillat, Phys. Rev. A 93, 013410 (2016). 